\title{
ArcheoSciences
}

Revue d'archéométrie

$34 \mid 2010$

Varia

\section{Le plomb chez Vannoccio Biringuccio, De La Pirotechnia (1540)}

Lead in the major work by Vannoccio Biringuccio - 1540

Patrick Clerc

\section{(2) OpenEdition}

1 Journals

Édition électronique

URL : https://journals.openedition.org/archeosciences/2683

DOI : $10.4000 /$ archeosciences. 2683

ISSN : 2104-3728

Éditeur

Presses universitaires de Rennes

Édition imprimée

Date de publication : 10 avril 2010

Pagination : 115-119

ISBN : 978-2-7535-1407-2

ISSN : 1960-1360

\section{Référence électronique}

Patrick Clerc, "Le plomb chez Vannoccio Biringuccio, De La Pirotechnia (1540) », ArcheoSciences [En ligne], 34 | 2010, mis en ligne le 11 avril 2013, consulté le 28 janvier 2022. URL : http://

journals.openedition.org/archeosciences/2683 ; DOI : https://doi.org/10.4000/archeosciences.2683

Article L.111-1 du Code de la propriété intellectuelle. 


\title{
Le plomb chez Vannoccio Biringuccio, De La Pirotechnia (1540)
}

\author{
Lead in the major work by Vannoccio Biringuccio - 1540
}

\author{
Patrick CLERC*
}

\begin{abstract}
Résumé : En 1540 est imprimé à Venise le premier livre traitant de toute la métallurgie. Il s'agit de l'ouvrage du signor Vannoccio Biringucio De la pirotechnia. Cet ouvrage paraît un peu avant le célèbre De Re Metallica de Giorgius Agricola (1556). Abordant en dix livres de nombreux sujets et questions pratiques ou métaphysiques, l'ouvrage de Vannoccio Biringuccio fait une place de choix au plomb dont on trouve des mentions plus ou moins développées dans la plupart des livres composant cette "Pirotechnia».
\end{abstract}

\begin{abstract}
In 1540 the first book dealing with metallurgy as a whole was printed in Venice. This is the work of Signor Vannoccio Biringucio entitled De la pirotechnia. The work was published shortly before the renowned De Re Metallica by Giorgius Agricola (1556). Addressing numerous subjects and practical or metaphysical questions in ten books, the work by Vannoccio Biringuccio gives a choice place to lead which is mentioned and described more or less extensively in most of the books included in "Pirotechnia".
\end{abstract}

Mots clé : Biringuccio, Pirotechnia, plomb, traité de métallurgie.

Key words: Biringuccio, Pirotechnia, lead, dealt of metallutgy.

En 1540 est imprimé à Venise le premier livre traitant de toute la métallurgie. Il s'agit De La Pirotechnia, écrit par Vannoccio Biringuccio (Sienne 1480-† Rome 1537) et édité à titre posthume par Curzio Navo chez Ventutino Roffinello (fig. 1). Publié 16 ans avant le De Re Metallica d'Agricola, cet ouvrage va rapidement inspirer les concurrents directs qui ne vont pas tarder à éditer de leurs côtés des œuvres parfois tout aussi significatives et masquer ainsi peu à peu le travail du métallurgiste italien souvent sans lui en reconnaittre la paternité. Aujourd'hui cependant, ce livre est considéré comme une œuvre fondamentale dans la littérature technologique qui prend place dans une tradition littéraire et scientifique alors en pleine mutation en osant imposer un style, une organisation systématique du sujet qui la différencie des innombrables petits traités techniques spécifiques

* Inrap.

rec. Oct. 2009; acc. May 2010 ou de recettes et de secrets alchimiques, sans pour autant les ignorer.

Chapitre après chapitre, l'ouvrage aborde toutes les métallurgies, de la fabrication du petit caractère d'imprimerie en plomb (dont Vannoccio Biringuccio est le premier à définir la délicate méthode de fabrication), aux coulées monumentales de bronze, toutes aussi délicates, des cloches et des canons. Les autres arts du feu ne sont pas en reste puisque sont abordés aussi la chimie, la verrerie, la production de la céramique et même des feux d'artifice. Tous les métaux alors connus sont évoqués depuis la mine d'où ils sont issus et la préparation de ces matières premières, à l'objet semi-fini, toutes les étapes de la chaîne opératoire sont présentées.

Parmi ces métaux, il en est un qui semble particulièrement ordinaire sans être quelconque, qui s'avère pourtant précieux 


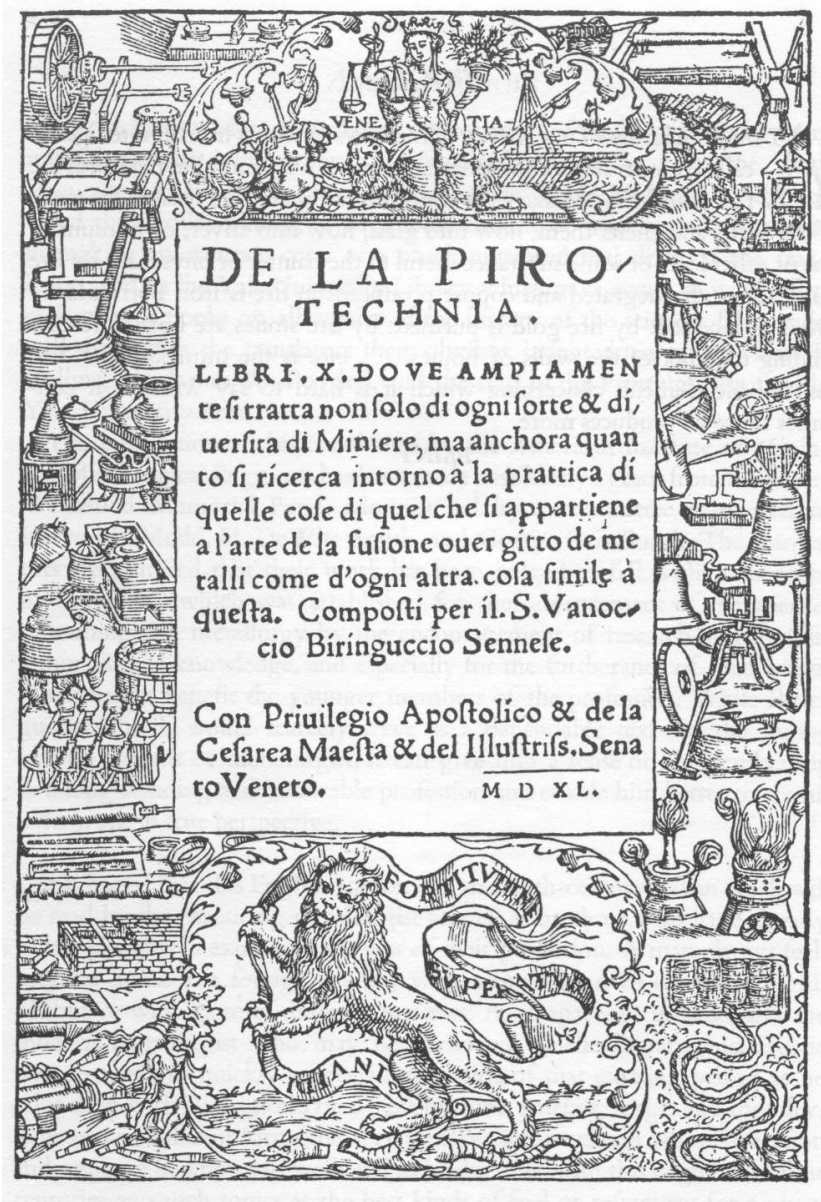

Figure 1 : Frontispice de l'édition originale de 1540 du De La Pirotechnie de Vannoccio Biringuccio.

Figure 1: Frontispiece of the original edition.

tant il est partout présent, parfois intimement lié aux autres. Connu et travaillé depuis si longtemps, apparemment si facile à travailler que même les alchimistes ont cru pouvoir le transformer en or. Il s'agit évidemment du plomb $(\mathrm{Pb})$.

Comment Vannoccio Biringuccio considère-t-il ce métal qui, d'un côté est sujet à tant de spéculations de la part des alchimistes et de l'autre, reste si présent et incontournable dans la métallurgie qu'il pratique chaque jour?

Il faut se plonger dans son livre pour trouver une partie de la réponse car les informations sont nombreuses, parfois discrètes. Il est indispensable de considérer l'ouvrage dans son environnement historique et son organisation interne pour apprécier la place tenue par le métal de saturne dans cet univers textuel.

D'entrée, le titre est éloquent : "De la Pyrotechnie, en dix livres, où l'on traite amplement non seulement de toutes les sortes et divers minerais, mais encore comment l'on recherche autour de la pratique des choses ${ }^{1}$ qui appartiennent à l'art de la fusion ou fonte des métaux ainsi que des choses semblables à cela ${ }^{2}$. " Il s'agit bien de la pyrotechnie comme "Art du feu », et non pas simplement de la fabrication des feux d'artifice comme on pourrait le comprendre aujourd'hui et comme cela a souvent été interprété.

Écrite en un italien populaire ${ }^{3}$, dense et abrégé, la Pirotechnia n'est pas facile à aborder. Un exemple d'abréviation, parmi tant d'autres, pour le mot " plomb " qui est régulièrement abrégé ainsi : " plôb ». Très souvent les $\mathrm{N}$ et les $\mathrm{M}$ sont réduits sous la forme d'un tiret, ou d'un accent circonflexe placé sur la lettre qui précède sa position. La ponctuation est occasionnelle et les phrases parfois très longues. L'ensemble est donc laborieux à lire et à comprendre, d'autant plus si on ne fait qu'aborder un passage ciblé. Les pages de texte forment des blocs impressionnants de 42 lignes de 65 caractères environ. Les illustrations, au nombre de 84 , réalisées à partir de gravures sur bois, sont probablement de la main même de l'auteur. Elles sont parfois difficiles à interpréter et ne valent pas les centaines de planches du $D e$ Re Metallica.

Comme pour la première édition de 1540, Curzio Navo est à l'origine des deuxième et troisième éditions publiées respectivement à Venise en 1550 et $1558^{4}$. En 1559 apparaît une nouvelle édition, un peu curieuse, dans un format nettement plus petit ${ }^{5}$. Il faut attendre $1678^{6}$ pour connaitre une nouvelle édition, elle aussi au format de poche. Une édition partielle en $1914^{7}$ initiée par le savant italien Aldo Mieli reste malheureusement sans suite. Pour finir, un fac simile de la première édition est publié en 1977, par Adriano Carugo.

La Pirotechnia rencontra rapidement du succès en Italie, mais également à l'étranger au regard des nombreuses traductions. C'est en France qu'est réalisée la première traduction intégrale, par "feu maître Jacques Vincent " qui sortira de l'imprimerie parisienne « chez Claude Fremy à l'enseigne

1. On pourrait remplacer le terme de « chose " par : méthode, procédé, pratique, moyen, etc.

2. De La Pirotechnia, Libri X, dove ampiamente si tratta non solo di ogni sorte \& diversita di miniere, ma encora quanto si ricerca intorno a la prattica di quelle cose di quel che si appartiene a l'arte de la fusione over gitto de metalli come d'ogni altra cosa simile a questa; in éd. 1540 Venezia.

3. Vulgare.

4. Respectivement imprimées, in quarto chez Giovan Padoano avec une dédicace à Guidotto Napio Boemo, et chez Comin da Trino avec une dédicace à l'Archidiacre de Ragusa, Mario Caboga.

5. In ottavo, imprimée toujours à Venise chez Gironimo Giglio.

6. À Bologne, in ottavo.

7. Aldo Mieli, (Livorno 1879 - Buenos Aires 1950), éditée à Bari; nombreux articles du même auteur : ISIS Vol. II, n 5, 1914; La Storia della Scienza in Italia, 1916; La Miniera Italiana, n² 2, 1917; Pagine di storia della chimica, 1922, etc. 
St. Martin », en 1556, sous le titre : "La Pirotechnie, ou Art $d u$ feu, contenant dix livres, auxquels est amplement traicté de toutes sortes et diversité de minières, fusions et séparations des métaux..." Une deuxième édition de cette traduction paraît en 1572, à la même enseigne. Une dernière édition intégrale française verra le jour à Rouen, en 1627, chez Jacques Cailloue. En 1856, de larges extraits seront traduits par Rieffel pour former un petit "Traité de la fabrication des bouches à feu de bronze au XVI siècle en Italie " ". Deux traductions partielles, en anglais, sont incorporées, l'une dès 1555 à Londres par Richard Eden dans son recueil "The Decades of the New Worde", et l'autre dans un des premiers traités sur l'art de la guerre "Certain Waies for the Orderyng of Souldiers in Battelray" de Peter Whitehorn en 1560. Une traduction partielle, en espagnol, est insérée dans le "De Re Metallica " de Bernardo Perez de Vargas publié à Madrid en 1569, sans pour autant reconnaittre l'origine des passages empruntés à Biringuccio. Concernant les traductions allemandes, il faut attendre 1925 pour trouver une remarquable traduction complète et commentée par Otto Johannsen "Biringuccio Pirotechnia, Ein Lehburch und des Artilleriewesens aus dem 16. Jahrhundert ${ }^{9}$ ». Plus récemment, deux éditions complètes, en anglais, sont réalisées à New York en 1942 et $1959^{10}$.

La Pirotechnia est constituée de dix livres subdivisés en chapitres. La présence d'un sommaire détaillé affiche, d'entrée, la volonté d'une œuvre méthodique et ordonnée. Le premier livre traite de tous les minerais métallifères alors connus, et débute avec un prélude qui présente quelques règles générales sur la recherche des gisements et sur la façon de creuser les mines ${ }^{11}$, avec les nouvelles méthodes, naissantes en Italie ${ }^{12}$, pour l'abattage, le boisage des galeries et la sécurité des mineurs. Au quatrième chapitre, dans l'ordre de préférence après l'or, l'argent et le cuivre, arrive le chapitre dédié au plomb ${ }^{13}$. Vannoccio Biringuccio connaît bien ce métal et le dit souvent mêlé à de grandes impuretés, et qu'il est appelé de ce fait " métal imparfait \& lépreux \& peu fixe " comme il le démontre par "fusion facile ". Mais l'auteur italien ajoute tout de suite qu'il est tenu " d'avoir grande

8. \$ III du livre V, \$\$ Ier, III, V, VI, VII, VIII et VIII bis, une partie du $\mathrm{X}, \mathrm{XI}$ du livre VI, $\$ \$$ Ier, V, VI et VIII du livre VII de la Pirotechnia de 1540, imprimé chez J. Corréard, Paris, in ottavo, 124 p. et pl.

9. Éd. Verlag von Friedr. Vieweg \& Sohn Akt.-Ges., xvi-544 p., Brauschweig, 1925.

10. Cyril Stanley-Smith, "The pirotechnia of V. Biringuccio ».

11. Les mines sont appelées "cave " par Biringuccio, synonyme de carrière. Les "miniere " sont les minerais.

12. R. Vergani, Progressi e ritardi nelle techniche venete : l'estrazione mineraria e la metallurgia dal XV al XVIII secolo, in Atti dell'Istituto Veneto di Scienze, Lettere ed Arti, CXLIX (1990-1991), IVSLA, Venezia, 1991, p. $209-237$.

13. Folio 13 de l'édition originale de 1540. obligation", car sa nature lui donne "dans son âme une si grande soif et désir d'avoir or et argent en grande quantité". Il précise encore que "sans son moyen il n'est pas possible de tirer du cuivre l'argent et l'or». Parmi les nombreuses utilisations possibles, dans les domaines autres que la métallurgie, l'auteur ne mentionne que les médecins "qui en beaucoup de médecines s'en servent " et en cosmétique chez certaines dames pour "l'art se dispose à certaine blancheur...en telles sorte qu'elles trompent la saincte vue des hommes » avec l'application sur la peau de céruse. Cette substance, appelée aussi "blanc de plomb » ou "blanc de Saturne », est un carbonate de plomb réduit en poudre. Même s'il existe à l'état naturel, il semble qu'il n'ait pas été utilisé comme pigment, et qu'il ait très tôt été manufacturé. Le procédé de fabrication, assez simple, consiste à exposer aux vapeurs de vinaigre (acide acétique), contenu dans un récipient en argile, des feuilles de plomb pendant plusieurs semaines. Le plomb métallique se convertit en une croûte blanche que l'on sépare du métal non transformé. Les croûtes sont ensuite lavées et broyées pour être utilisées. C'est dans ce chapitre que sont mentionnés les "feu de rabat ou de réverbération " que Biringuccio est un des premiers à décrire clairement.

Le deuxième livre a pour objet les substances non métalliques " ni tout à fait pierre, ni tout à fait métal " comme l'argent vif, c'est-à-dire le mercure, le soufre, l'antimoine, la marcassite, la calamine, l'arsenic... de l'azur, du cristal et d'autres pierres précieuses. Il termine par un dernier chapitre sur la fabrication du verre. Dans le quatrième chapitre dédié à la marcassite et ses couleurs sombres, Biringuccio compare une matière noire qu'il dit " comme nielle ou de couleur plombine ${ }^{14}$ ". Il reviendra dans le neuvième livre au chapitre sur le travail de l'orfevvre sur la composition de cette substance.

Le troisième livre est l'un des plus importants de l'œuvre, car il traite non seulement des essais menés sur les minerais, en particulier pour l'argent et l'or ${ }^{15}$, de leur préparation à la fonte, mais aussi de la séparation du plomb du cuivre grâce à la liquation, de l'or et de l'argent des autres métaux à travers la coupellation. Pour la fabrication des petites coupelles destinées à éprouver les minerais, Biringuccio recommande notamment l'utilisation de cendres d'os de pattes de chevaux, d'âne ou de mulet, bien calcinées, lavées à l'eau, recuites, pulvérisées et bien séchées (fig. 2). Quand il parle des moyens de "molifier" les matières, il indique qu'il peut être utile d'ajouter, comme fondant, du marbre, du verre pilé ou de la "ghetta di piombo». Ce terme désigne ce que nous appelons aujourd'hui la litharge, cet oxyde de plomb

14. Folio $28 \mathrm{v}^{\circ}$.

15. Folio 45 et suivant. 


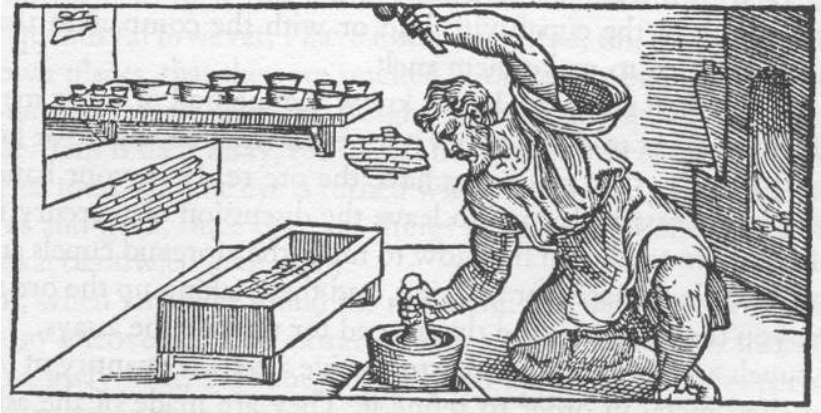

Figure 2 : Figure $\mathrm{n}^{\circ} 34$ de l'édition originale de $1540 \mathrm{du}$ De La Pirotechnie de Vannoccio Biringuccio, avec représentation de la préparation des coupelles pour éprouver les métaux.

Figure 2: Figure 34 of the original edition.

issu de la coupellation, dont il parle aussi dans le septième chapitre, mais surtout au neuvième. La traduction française, de 1556, utilise aussi le mot de "ghetta " sans le traduire. Dans le fameux septième chapitre, où il décrit les détails de la coupellation, il évoque alors le moment subtil de "l'éclair d'argent", où il faut "voir parmi les flammes du bois que le plomb semblera être de couleur azurée, puis après noire, et en un instant deviendra clair et luisant comme une étoile ${ }^{16}$ ".

Le quatrième livre est relativement court, car il décrit la séparation et l'affinage de l'or à partir de l'argent grâce notamment aux acides. Le plomb n'entre pas en œuvre dans ces procédés, chez Biringuccio.

Le cinquième livre, encore plus concis, en quatre chapitres, traite des alliages de l'or, de l'argent, du cuivre et, dans ce dernier chapitre, de l'affinité naturelle qu'ont le plomb et l'étain. Biringuccio parle des "marchants estrangers disent qu'il se doit allier avec le plomb ơ quil est meilleur à mettre en cuvre quand il tient 4 ou 6 livres pour cent, assurant qu'il est plus doux au marteau, plus fluide coulant et net au jeter ", c'est-à-dire à couler, mais que ce rapport ne lui est aucunement agréable. Il préfere ainsi utiliser l'étain "estant pure que mêlé", et que l'association du plomb n'est utile qu'à ceux qui veulent souder le cuivre et l'autre pour les maitres verriers qui désirent blanchir leurs vases ${ }^{17}$.

Le sixième livre est dédié aux grandes fontes en bronze de pièces d'artillerie et de cloches. Toute l'expérience et le savoir-faire du maitre fondeur vont s'expliquer en une quinzaine de chapitres. La numérotation de ces derniers est erronée dans le sommaire de l'édition originale, mais sans nuire au texte.

16. $\mathrm{f}^{\circ} 57:$ :[...] a un trato farsi come una stella chiaro \& lucido." 17. Folio $74 \mathrm{v}^{\circ}$.
Le septième livre décrit les divers types de fours utilisés pour fondre le métal. Là encore, l'expérience de l'auteur transparaît clairement à travers sa description du four à réverbération, et celles des pièces d'artilleries.

Le huitième livre traite de "l'arte picola del gitto " c'est-àdire de la fonte des objets artistiques et d'usage commun à l'aide de moules. Après les canons et les cloches, Biringuccio affine la préparation des moules devant recevoir les coulées les plus fines.

Le neuvième livre traite de plusieurs domaines touchant au travail des métaux, de l'art du forgeron, du chaudronnier, de l'étameur, du batteur d'or, sur la frappe des monnaies, sur l'art des potiers... Le premier chapitre est dédié à l'alchimie et aux "philosophes en action", comme il aime les appeler. La distillation de nombreuses substances est abordée et illustrée de plusieurs planches. Le quatrième chapitre parle du travail d'orfèvre, et notamment de l'élaboration du nielle. Le niellage est une technique d'orfevrerie qui consiste à appliquer le niello (du latin nigellus, noirci) pour le remplissage de petites cloisons laissées vides à cet effet sur les objets à décorer. Il s'agit, selon Biringuccio, d'un mélange d'une portion d'argent pur pour deux de cuivre et trois de plomb fin. Au septième chapitre, dédié au travail de l'étain, Biringuccio nous livre la première description détaillée du mode de fabrication des caractères d'imprimerie. En effet, la méthode par laquelle Gutenberg fabriquait ses caractères pour imprimer et publier, en 1455 , le premier livre nous est inconnu et il est singulier de devoir attendre 1540 pour lire dans La Pirotechnia cette description : "Les lettres pour imprimer les liures se font premièrement en composition de trois parties d'étain fin, une huitième partie de plomb noir et autant de marcassite et d'antimoine fondue et mélangé ensemble ${ }^{18} . . . "$

Le dixième et dernier livre est dédié aux arguments pyrotechniques, au sens strict. Biringuccio y décrit une série de mélanges incendiaires, de poudres, d'explosifs utiles en ces périodes troubles, de la façon de faire des boulets de canon, de la manière à faire sauter des mines souterraines ${ }^{19}$, et enfin des feux d'artifice et autres girandoles " pour faire l'allégresse dans les cités de Toscane ». Il termine son œuvre par un ultime chapitre, dédié au "feu qui consume et qui ne fait pas de cendre et qui pourtant est plus puissant que tous les autres feux ", c'est-à-dire de l'amour, de l'amour de son métier, de l'amour de sa vie.

Les nombreuses utilisations du plomb dans la métallurgie de Vannoccio Biringuccio qui transparaissent ça et là dans son traité dévoilent le côté incontournable de ce métal dans les procédés les plus classiques de son art.

18. "Le letere da stampare li libri", $\mathrm{f}^{\circ} 138 \mathrm{v}^{\circ}$ de l'éd DLP de 1540. 19. "De le mine sotterranee ", $f^{\circ} 165$. 
Ce métal, qui semble facile d'usage pour les apprentis métallurgistes, est facile à travailler et à moindre coût, facile à tester lors d'expériences diversifiées. Nombreux sont ceux qui vont tester leurs compétences techniques à lancer une opération sur le feu, peu nombreux sont ceux qui parviendront à comprendre les réactions provoquées. Ensuite, tout va dépendre de la qualité des interprétations et des théories qui en résultent, de la conscience scientifique de ces essayeurs. On pourrait dégager deux courants de résultats : d'un côté, la " culture alchimique " prendra le dessus pour aboutir aux théories visant avant tout à exploiter ces énigmes à des fins autres que scientifiques; de l'autre, le pragmatisme dominera et donnera lieu à d'autres questions, d'autres expérimentations et parfois à des résultats exploitables.

Vannoccio Biringuccio a toujours été très intrigué par les conceptions des alchimistes. Ne pouvant les ignorer, il les a parfois mesurées et éprouvées, les plus crédibles du moins. Il finira, comme quelques-uns, à estimer qu'il y avait deux alchimies : la sérieuse, qui est l'ancêtre de la chimie, sur laquelle il s'appuie régulièrement quand il parle de la distillation ou de la sublimation, par exemple, des acides ou du mercure; et l'alchimie mystique, ou magique, qui est celle partie à la recherche de la pierre philosophale, qui dit transformer le plomb en or, celle qu'il finira par rejeter. Dans de longs passages, il fustige ces "philosophes en action ", notamment en affichant clairement son scepticisme au sujet de cette idée. Il dit : « Non seulement je ne crois pas que l'on puisse le trouver, mais que jamais quelqu'un avec vérité ne l'a vue, encore que beaucoup de monde dit l'avoir vue ${ }^{20}$. "

Vannoccio Biringuccio considère dans son œuvre le plomb pour ce qu'il est, c'est-à-dire un métal utile à tant de procédés pratiques, au point d'en avoir "grande obligation", notamment pour exploiter ses qualités techniques lors de ses travaux métallurgiques. Ce métal, maintenant bien domestiqué, finira par devenir précieux, non pas par sa valeur pécuniaire, mais bien pour ses qualités physiques et techniques, pour son utilité pratique. Le seul intérêt intrinsèque et "spirituel " de ce métal, chez notre auteur, fut peut-être de lui avoir servi à se dissocier clairement des alchimistes dans son livre, quittant ainsi définitivement, pour sa part, le caractère magique et religieux de cette science.

\section{Bibliographie}

Biringuccio, V., 1540. De La Pirotechnia, Libri X, dove ampiamente si tratta non solo di ogni sorte \& diversita di miniere, ma encora quanto si ricerca intorno a la prattica di quelle cose di quel che si appartiene a l'arte de la fusione over gitto de metalli come d'ogni altra cosa simile a questa, Venezia, éd. Curzio Navo.

Mieli, A., 1914. Vannoccio Biringuccio e il metodo sperimentale, in Isis, $\mathrm{n}^{\circ} 2,1914$, p. 90-99.

Mieli, A., 1914. De la Pirotechnia. di Vannoccio Biringuccio, edizione critica sulla prima edizione, corredata di note, prefazioni, appendici ed indici, ed ornata dalle riproduzioni del frontespizio e delle 82 figure originali, Bari, éd. Societa Tipografica Editrice Barese.

Mieli, A., 1921. Vannoccio Biringuccio, in Gli scienziati italiani dall'inizio del medio evo ai nostri giorni, Vol. 1, Rome.

Stanley Smith, C. and Teach Gnudi, M., 1990. De la Pirotechnia. The Pirotechnia of Vannoccio Biringuccio, the Classic SixteenthCentury Treatis on Metals and Metallurgy, New York, Dover Publications.

Vergani, R., 1991. Progressi e ritardi nelle techniche venete : l'estrazione mineraria e la metallurgia dal XV al XVIII secolo, in : Atti dell'Istituto Veneto di Scienze, Lettere ed Arti, CXLIX (19901991), IVSLA, Venezia, p. 209-237.

20. Cf. $\mathrm{f}^{\circ} 7 \mathrm{v}^{\circ}$ 
